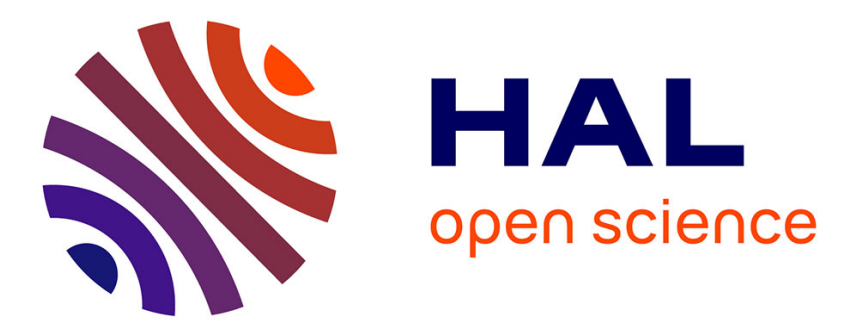

\title{
DVB-S2/DVB-RCS satellite system performance assessment for IFE and ATN aeronautical communications
}

Na Tao, Michel Bousquet, Alain Pirovano, José Radzik

\section{- To cite this version:}

Na Tao, Michel Bousquet, Alain Pirovano, José Radzik. DVB-S2/DVB-RCS satellite system performance assessment for IFE and ATN aeronautical communications. IWSSC 2006, International Workshop on Satellite and Space Communications, Sep 2006, Madrid, Spain. pp 170-175, 10.1109/IWSSC.2006.256017 . hal-01021787

\section{HAL Id: hal-01021787 \\ https://hal-enac.archives-ouvertes.fr/hal-01021787}

Submitted on 9 Sep 2014

HAL is a multi-disciplinary open access archive for the deposit and dissemination of scientific research documents, whether they are published or not. The documents may come from teaching and research institutions in France or abroad, or from public or private research centers.
L'archive ouverte pluridisciplinaire HAL, est destinée au dépôt et à la diffusion de documents scientifiques de niveau recherche, publiés ou non, émanant des établissements d'enseignement et de recherche français ou étrangers, des laboratoires publics ou privés. 


\title{
DVB-S2/DVB-RCS satellite system performance assessment for IFE and ATN aeronautical communications
}

\author{
$\mathrm{Na} \mathrm{Tao}{ }^{1}$, Michel Bousquet ${ }^{2}$, Alain Pirovano ${ }^{1}$ and José Radzik ${ }^{2}$ \\ ${ }^{1}$ Ecole Nationale de 1'Aviation Civile (ENAC) \\ 7 avenue Edouard Belin, BP 4005 \\ 31055 TOULOUSE Cedex 4, France \\ 2 SUPAERO \\ 10 avenue Edouard Belin, BP 54032 \\ 31055 TOULOUSE Cedex 4, France
}

na.tao@recherche.enac.fr, michel.bousquet@supaero.fr, alain.pirovano@enac.fr, jose.radzik@supaero.fr

\begin{abstract}
This paper presents the PhD work conducted by Na TAO. The director of this thesis is Professor Michel BOUSQUET. The study is supervised by Alain PIROVANO and José RADZIK. This PhD work has started January 2006 and will end in December 2008.

Two concurrent satellite systems are presently proposed for in-flight entertainment (IFE) communications, thus demonstrating the capability of satellites to provide multimedia access to users on board airplanes. At the same time, an increasing interest for the use of satellite communications for air traffic control has been motivated by the increasing load of traditional radio links, mainly in the VHF band. However, the development of a dedicated satellite system for ATC (Air Traffic Control) and ATN (Aeronautical Telecommunication Network) seems to be a long-term perspective. The objective of the presented system design is to provide both passenger application traffic (Internet, GSM) access and a high-reliability channel for aeronautical applications using the same satellite links. Due to the constraints in capacity and radio bandwidth allocation, very high frequencies (above $20 \mathrm{GHz}$ ) are considered here. The corresponding design implications for the air interface are taken into account and access performances are derived using a dedicated simulation model. Some preliminary results are shown in this paper to demonstrate the technical feasibility of such system design with increased capacity, more details and the open issues will be studied in the future of the thesis research.
\end{abstract}

\section{INTRODUCTION}

$\mathrm{D}_{\mathrm{E}}$ URING the recent years, IFE (In-Flight Entertainment) has become one of the hot topics in the communications world and seems to be one of the winning factors for airlines and aircraft industry. Supplied services IFE can be VPN access, e-mail, real-time video and web browsing. Thanks to the satellite communications systems, the validity of service IFE has been proven. The two major aeronautical manufactures promote the IFE systems in recent years: Connexion by Boeing and OnAir by Airbus.

At the same time, an increasing interest for the use of satellite communications for ATC (Air Traffic Control) has been motivated by the increasing load of traditional radio links, mainly in the VHF band. However, the development of a dedicated satellite system for ATC and ATN (Aeronautical Telecommunication Network) seems to be a long-term perspective. In our design, the satellite link acts as a supplementary access network for ATC/ATN communications and when available can be chosen as an alternative to VHF or HF links.

The objective of this thesis work focus on a network system design using the same satellite link to provide both Internet-like access and a high-reliability channel for aeronautical applications. The rest of this paper is organized as follows. Section 2 provides an overview of the system architecture which bases on the present hypothesis. Section 3 focuses on the study of the resource management; the main problem and the solution will be introduced. Section 4 describes the system design according to the system architecture and definition. Section 5 shows some preliminary results which demonstrate the technical feasibility. Section 6 proposes the future work. Finally, conclusion is provided in section 7 .

\section{SYSTEM DEFINITION}

The system presented in this paper relies on the use of a multimedia satellite access network using the ETSI DVBS2/DVB-RCS architecture [1] [2].

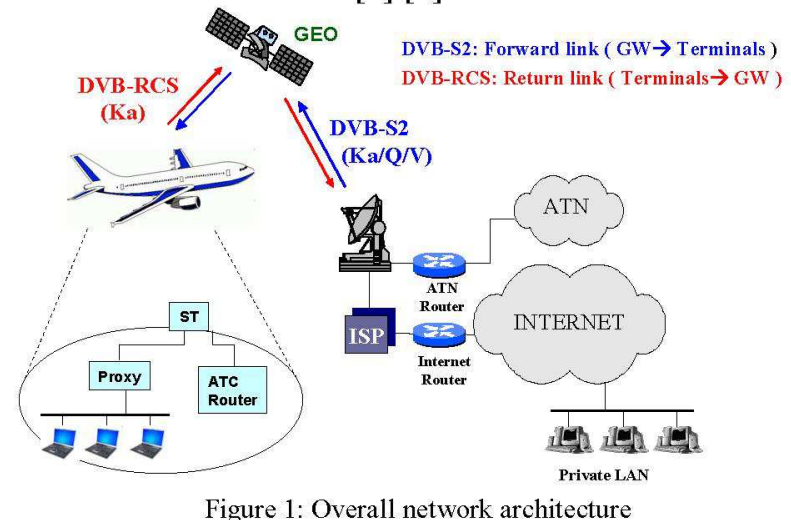

The reference model for a DVB-S2/DVB-RCS network is 
shown in Figure 1. Some present hypotheses of such system are mentioned as follows:

- Band frequency: the forward link (gateway to terminal) uses $\mathrm{Ka}$ or $\mathrm{Q} / \mathrm{V}$ frequency and the return link (terminal to gateway) uses Ka frequency

- Satellite broadband services: DVB-S2 standard is applied in the forward link and DVB-RCS standard for the return link

- Satellite: a GEO bent pipe satellite is chosen for our system design. No hypothesis is made on the service zone coverage. Routing on board the satellite in not needed and a regenerative payload could be considered for link budget enhancement and capacity increase

- Topology: the network topology is a classical star network with the GW station as a Hub.

DVB-S2 (Digital Video Broadcasting - Satellite 2) is the second-generation specification for satellite broadcasting. It offers a large capacity by using very high frequencies and can carry either unicast or broadcast traffic (like TV information programs). DVB-RCS (Digital Video Broadcasting - Return Channel by Satellite) is designed by adding a return channel to the DVB-S2 and gives an interactive way for the services via satellite.

\section{RESOURCE MANAGEMENT IN DVB-S2/DVB-RCS NETWORK}

Due to the use of high frequencies, encountered fades should be very high. Figure 2 shows the encountered fades at Ka band. To compensate such destroying fades, FMT (Fade Mitigation Techniques) must be considered. Obviously, the system can not be designed with a constant link margin; otherwise the waste of capacity would be excessively high.

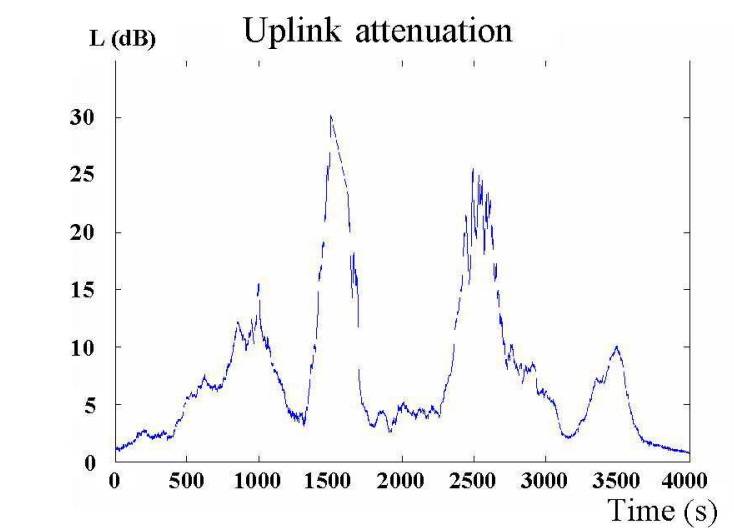

Figure 2: Uplink fade event. Example of deep fade event (convective rain)

DVB-S2 access technique is TDMA with an embedded technique for fade mitigation based on FEC management. Data are sent in blocks of constant size (in number of coded bits) and modulation and coding are adapted to the actual propagation conditions observed on each individual link. It should be noticed that DVB-S2 relies on carrier to noise and interference ratio measurements (CNI) that are carried from terminal to gateway using the DVB-RCS return link. The $\mathrm{CNI}$ reports have been introduced in the last version of the DVB-RCS standard [1].

The resource management of DVB-RCS links is more challenging due to the MF-TDMA access. The standard does not explicitly introduce fade mitigation techniques; on the DVB-RCS link, the useable techniques are:

- UPC (Uplink Power Control): transmitter power is increased to counteract fade or decreased when more favorable propagation conditions are recovered to optimize satellite capacity

- DRA (Data Rate Adaptation): nominal data rates are used under clear sky conditions (no degradation of the service quality with respect to the system margin), whereas reductions is introduced according to fade levels

- ACM (Adaptive Coding and Modulation) [3]: using the different coding and modulation modes on the different carriers to match impairments due to propagation conditions.

In DVB-RCS, the resource management process DAMA (Demand Assignment Multiple Access) must take into account the required mode for a given terminal in order to choose the right carrier for resource allocation. The considered modulations are QPSK (the DVB-RCS present definition), BPSK and 8PSK (extension of available modes in the standard). Coding rates (using Turbo-coding) range from $1 / 3$ to $6 / 7$.

The radio design on the return link is proposed following three steps:

1) In the first step, calculate the classical link budget in the case of clear sky

2) Then, analyse the link budget by considering the rain attenuation and calculate the overall available margin with FMT

3) Finally choose the used modes

In the considered design, the symbol rate is set to 683 ksymbol/s and the clear sky mode uses 8-PSK with a $1 / 2$ coding rate. The corresponding data rate is $1024 \mathrm{kbit} / \mathrm{s}$. The clear sky link budget includes a $2 \mathrm{~dB}$ static margin that is needed mainly because of scintillation (this very fast fade variation is noticeable at $\mathrm{Ka}$ frequencies and can be considered as unpredictable. The variance of the phenomenon can however be modelled for margin determination). The most robust mode uses BPSK with a $1 / 3$ coding rate, the symbol rate is then $170 \mathrm{ksymbol} / \mathrm{s}$ (the reduction of symbol rate (DRA) gives an additional $6 \mathrm{~dB}$ margin).

The margin given by the FMT design is summed up in Table 1. 


\begin{tabular}{|c|c|c|}
\hline Clear sky mode & $\begin{array}{c}\text { 8-PSK } \\
1 / 2\end{array}$ & Req. Es/No $8.7 \mathrm{~dB}$ \\
\hline Most robust mode & $\begin{array}{c}\text { BPSK } \\
1 / 3\end{array}$ & Req. Es/No $-1.5 \mathrm{~dB}$ \\
\hline \multicolumn{2}{|c|}{ ACM Margin } & $10.2 \mathrm{~dB}$ \\
\hline \multicolumn{2}{|c|}{ DRA Margin } & $6 \mathrm{~dB}$ \\
\hline \multicolumn{2}{|c|}{ Uplink PC margin } & $19.2 \mathrm{~dB}$ \\
\hline \multicolumn{2}{|c|}{ Total FMT margin } &
\end{tabular}

Table 1: FMT Margin

The margin provided by FMT can be related to the availability of the link. Using the ITU model for long term rain fade prediction [4], the availability is $99.9 \%$ of the time. It should be noticed however that the typical rain fade event shown on figure 2 (storm with convective rain) has been synthesized considering a fixed land terminal. In the case of aeronautical communications, the movement of the aircraft should be taken into account. Knowing that convective rain cells have an average diameter of the order of $3 \mathrm{~km}$, the occurrence of the maximum loss will be less and the obtained availability is certainly higher.

\begin{tabular}{|c|c|c|c|c|}
\hline Mode & $\begin{array}{l}\text { Modulation } \\
\text { and coding }\end{array}$ & Req. Es/No & Symbol Rate & Data rate \\
\hline 0 & BPSK $1 / 3$ & $-1.5 \mathrm{~dB}$ & $170 \mathrm{ks} / \mathrm{s}$ & $56 \mathrm{kbit} / \mathrm{s}$ \\
\hline 1 & BPSK $1 / 3$ & $-1.5 \mathrm{~dB}$ & $683 \mathrm{ks} / \mathrm{s}$ & $228 \mathrm{kbit} / \mathrm{s}$ \\
\hline 2 & QPSK 1/2 & $4.5 \mathrm{~dB}$ & $683 \mathrm{ks} / \mathrm{s}$ & $683 \mathrm{kbit} / \mathrm{s}$ \\
\hline 3 & QPSK 2/3 & $6.9 \mathrm{~dB}$ & $683 \mathrm{ks} / \mathrm{s}$ & $910 \mathrm{kbit} / \mathrm{s}$ \\
\hline 4 & 8PSK $1 / 2$ & $8.7 \mathrm{~dB}$ & $683 \mathrm{ks} / \mathrm{s}$ & $1024 \mathrm{kbit} / \mathrm{s}$ \\
\hline
\end{tabular}

From the previous results, 5 modes are selected as presented in Table 2. The modes have been chosen with a constant symbol rate at the exception of mode 0 . This means that the carriers occupy a constant bandwidth; the mode management is hence easier, since the mode of a given carrier can be modified without any change of the frequency plan (DVB-RCS provides adequate signaling for frequency plan reconfiguration). Mode 0 should be considered as a rescue mode that has been introduced in order to provide a high availability for ATC traffic, it should be noticed that the obtained data rate $(56 \mathrm{kbit} / \mathrm{s})$ is of the same order than using VDL Mode 2 link (31.5kbit/s).

\section{SYSTEM DESIGN}

The hypothesis in the considered system is that the performance objectives for the multimedia access and the aeronautical services have to be addressed separately. Very short outages (of the order of one superframe, e.g. $100 \mathrm{~ms}$ ) are not detrimental for the Internet access, however some services need good performances in terms of transfer delay and delay variation (voice services for example). On the contrary, the link for aeronautical services must be compliant with the performance requirements issued by ICAO [5] and EUROCAE WG 53 [6]. These performances are given as time constraints (Maximum transaction duration ETRCP, duration for $95 \%$ of transactions TT95), availability (continuity CRCP, availability ARCP) and data transfer liability (integrity IRCP). In this case, priority must be set on availability and not capacity optimization, whereas time constraints are rather loose compared to Internet access (acceptable delays are of the order of 10 seconds). The return link access terminal will thus have to manage data flows with different requirements.

We propose here a simplify structure of the terminal which is presented in Figure 4

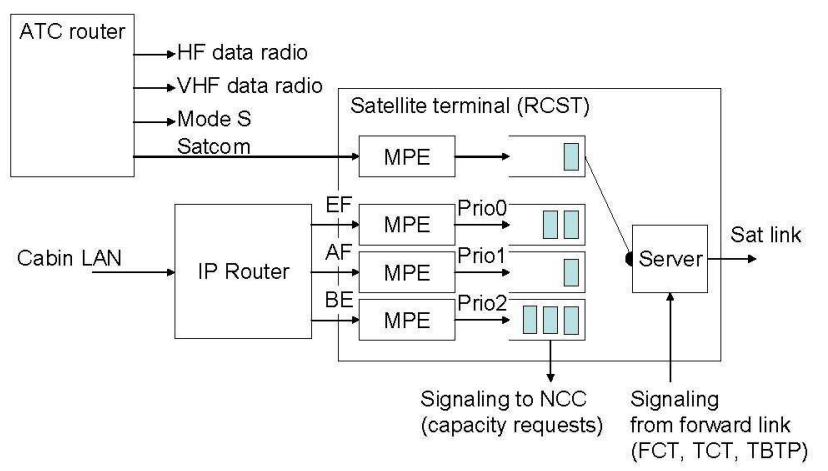

Figure 4: structure of the on-board satellite access terminal

The terminal accepts data on four SAPs (service access points). The first one is used by ATC traffic; the remaining three SAPs are dedicated to IP traffic. The hypothesis is made that multimedia traffic has been classified in the IP router with a DiffServ policy; for each IP datagram, the corresponding SAP is determined on the basis of the DiffServ code point label. Then the incoming traffic is segmented in MPEG packets using ULE and stored in queues associated to the different priority levels. The satellite terminal determines the needed capacity on the basis of queue monitoring; this capacity is translated in capacity requests according to the DVB-RCS signaling formats, either rate based or volume based requests.

The resource management in the gateway has been adapted in order to take into account the requirements for the two main services. Slots allocation in the DVB-RCS MFTDMA is made using three parameters: CRA (Constant Rate Allocation), which is a fixed allocation, available permanently to a satellite terminal whatever it is used; RBDC (Rate Based Dynamic Capacity) and VBDC (Volume-Based Capacity Requests) which are dynamically allocated on request of the ST. The allocation process uses a standard DAMA algorithm (allocation of CRA first, then RBDC requests and finally VDBC requests). The resulting allocation is sent to the terminals within the TBTP (Time Burst Time Plan).

Because of the long signaling loop $(500 \mathrm{~ms}$ are needed between the capacity request send time and the corresponding TBTP reception), on-board the airplane, a server is needed in order to send data on the satellite link according to a priority-based service policy. A "superpriority" is given to ATC queue, and then the three remaining queues are served with a WRR (Weighted Round 
Robin) service policy. It means that the ATC traffic "steals" capacity from multimedia traffic when needed in order to get the highest possible availability. This aspect is very important in the case the terminal needs to use FMT mode 0 , since the available data rate is very low and does not permit to maintain a correct service for multimedia applications, the link is then used mainly for ATC traffic.

\section{PRELIMINARY SIMULATION RESULTS}

A simulation platform has been developed according to the presented design using the OPNET Modeler software (Opnet Technologies Inc.).

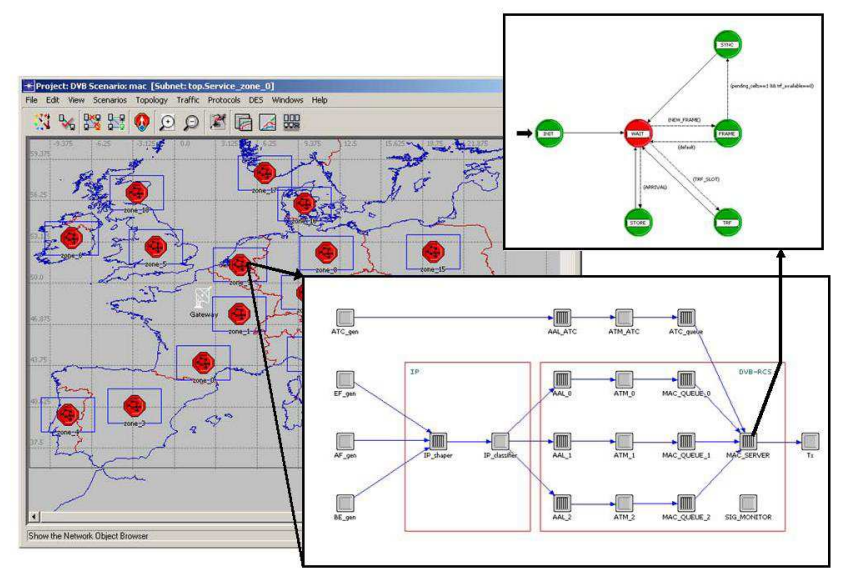

Figure 5: OPNET simulation model

The simulation model which is shown in figure 5 encompasses 50 terminals and one gateway corresponding to the traffic load expected in one satellite beam and a given superframe ID group (the simulated carriers represents a subset of the carriers in a $25 \mathrm{MHz}$ repeater). Within each terminal, 4 traffic sources are activated with different characteristics for each priority level. The ATC traffic is made of packets with a size uniformly distributed between 64 and 256 bytes and a mean arrival time of $90 \mathrm{~s}$. The EF traffic source corresponds to G.729 VoIP codecs. The AF traffic source represents an rt-VBR service (packets are also IP datagrams). The BE traffic source models bulk data transfer with both exponential laws for packet sizes and inter-arrival times, as for example FTP sessions. Each airplane can be considered as a LAN (Local Area Network) and the aggregate traffic obtained from the sources should be representative of a typical LAN.

When terminal is concerned by very deep fades, outages occur when Es/No (resp. Eb/No) becomes lower than the minimum required level. Figure 6 illustrates the behavior of such system in deep fade. ACM modes are chosen in order to maintain the link quality to the detriment of the useable data rate.

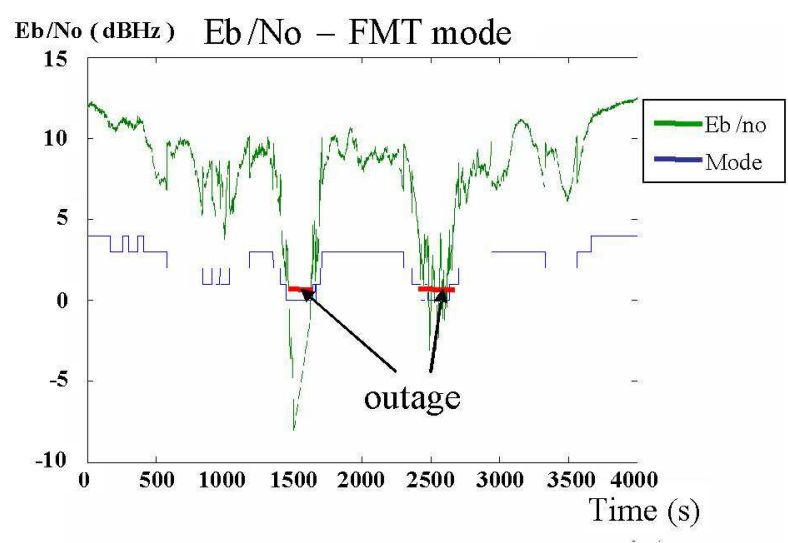

Figure 6: System in deep fade, ACM activation

The choice of the ACM mode relies on measurements made by the terminal and transmitted to the gateway (these measurements are needed in any case for the working of DVB-S2 and related signaling flows have been defined in version 1.4.1 of the DVB-RCS standard). The decision process for setting the current mode of a given terminal is conducted in the gateway (centralized mode) on the basis of the CNI reports. A compromise must be found between the following parameters:

- Probability of false detection (ACM mode not fitted to actual propagation conditions)

- Desired link quality (Packet Error Rate PER)

- Optimization of the resource utilization (chosen ACM mode should provide the best spectral efficiency)

From the CNI reports, the choice of the ACM mode is made with a margin that takes into account the unpredictable part of the fade (mainly scintillation at Ka-band), the uncertainty on the CNI measurement and the wanted probability of false detection. Determination of margin is a tricky task with a direct impact on capacity; simulations as presented below have been used to get the best fit.

In order to simulate the propagation conditions, terminals determine the present attenuation and required FMT mode thanks to synthesized time series [7]. However, in order to get reasonable simulation durations, terminals can be simulated either in clear sky mode or with simplified time series (only commutation times from one FMT mode to the next are simulated) or with the complete time series (attenuation is determined every second). Figure 7 shows the transition between FMT modes for one terminal. 


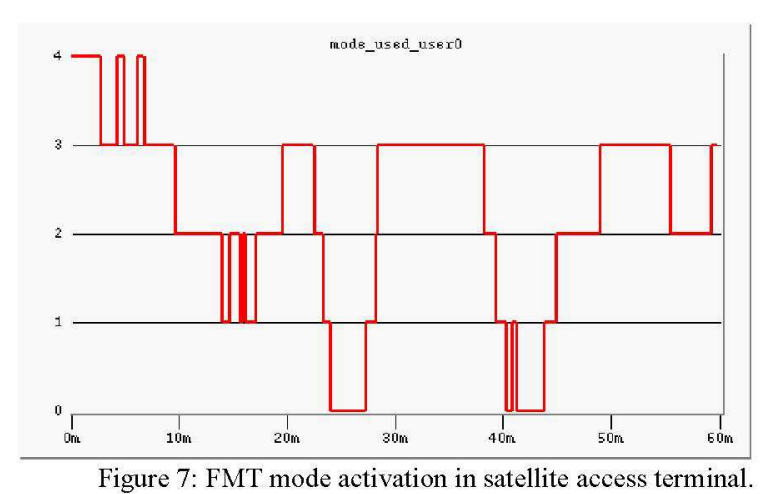

Upon reception of radio bursts at the gateway, a recovery process is conducted to get embedded MPEG packets. An error model is then used and an error code is associated to each packet: 0 - packet properly received, 1 - packet with errors in header, 2 - packet with errors in payload, 3 packet lost (TRF burst no received). This model which is shown in Figure 8 allows for a study of the behavior of the system when switching from one mode to the next. Depending on the activation margin, losses can be observed on FMT mode transition. It is also possible to determine the actual outage periods when all packets are received with errors either in header or payload. The chosen activation margin, hence the FMT activation loop design, has proven robust enough to get a PER of $10^{-7}$ outside outage periods.

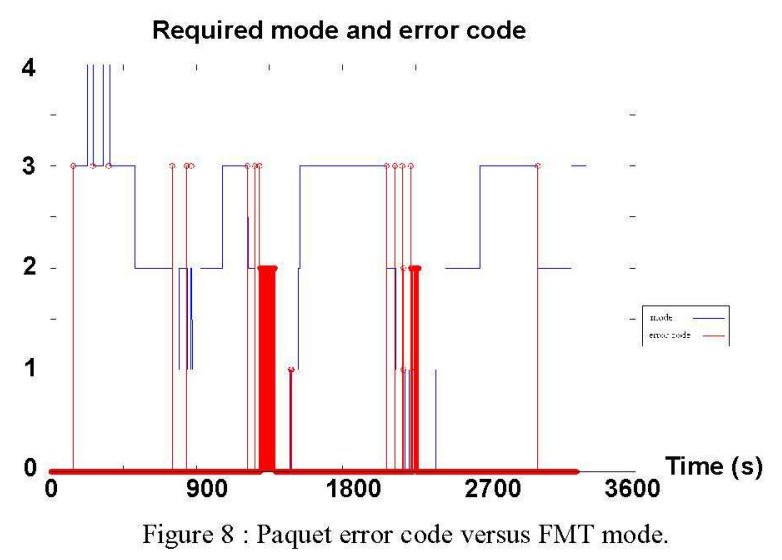

In the satellite access terminal, queuing delays have been monitored to check the correct behavior of the priorityoriented service policy. The probability density functions for queuing delays are plotted in figure 9 (outage periods are discarded).

Observed queuing delays are in conformance with the expected behavior. Priority 0 (corresponding to the EF traffic) gets shorter service times on average than the other priorities; the generated EF traffic is sent within a maximum of two superframes. Delays are spread over a wider range for priorities 1 and 2 . Because of the particular traffic shape and service policy, the ATC traffic gets the better service.

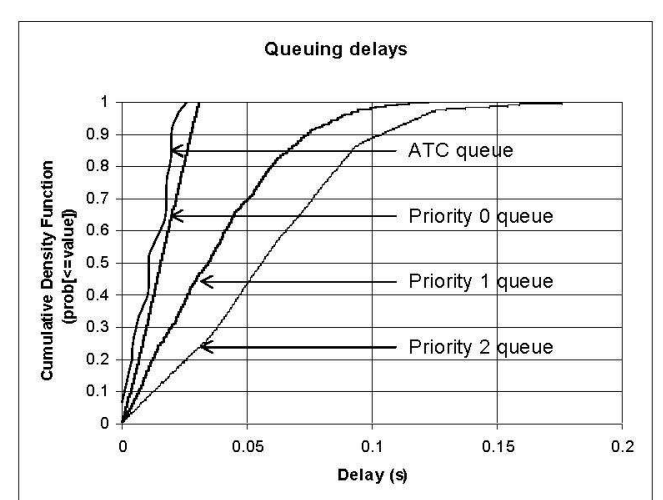

Figure 9: Queuing delays in satellite access terminal.

\section{PERSPECTIVES}

The complete study of such a complex system is out of reach for a $\mathrm{PhD}$ work, since many challenging design aspects must be considered: design of networks on board the aircraft, of the satellite payload... The actual scope of the $\mathrm{PhD}$ work is presented in Figure 10 and focuses on the architecture of the satellite terminal on board the plane.

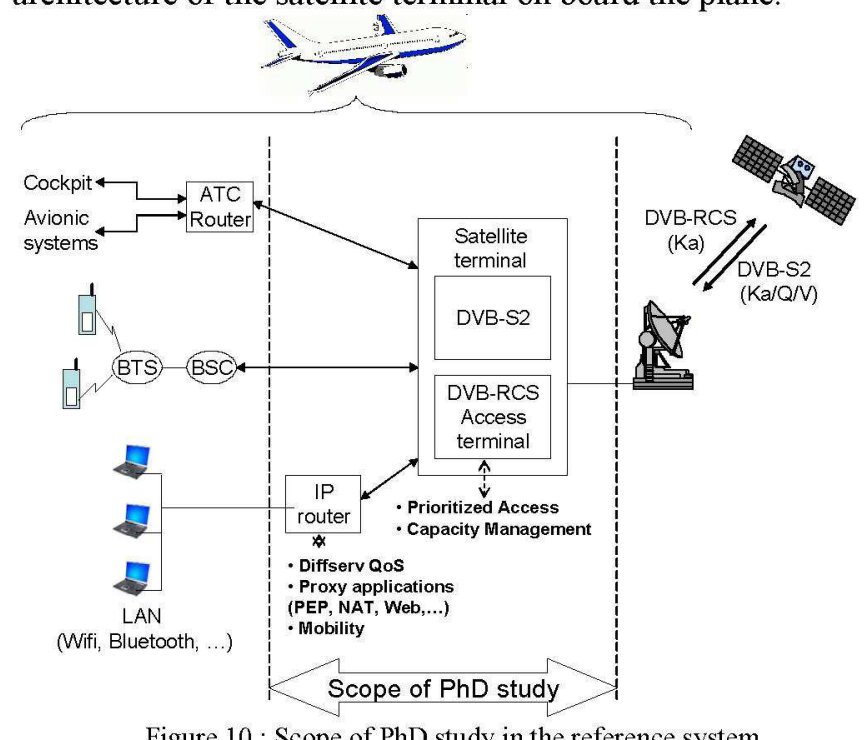

This means that some aspects must be considered as inputs from other research actions. As an example, we take for granted the feasibility of a wireless LAN within the plane and we will consider only the gathered traffic at the input of the access terminal. Another important aspect is the behavior of the satellite link with the activation of the FMT; here we will rely on results obtained thanks to studies conducted at SUPAERO with the contribution of other partners (ONERA, SatNex members).

The main research actions that will be conducted are listed here:

- $\quad$ ATC service study, traffic characterization and modeling, definition of required quality of service

- Topology and functionalities of on-board 
networks

- Study of solutions for improvement of performances of the transport layer

- Convergence in the network, multiplexing of IP, GSM and ATC traffic on single DVB links

- Traffic flow management and service policy in the medium access layer

- Mobility management in case of gateway handover or satellite handover

- Analysis of perceived service quality thanks to an emulation platform

\section{A. ATC service}

The ATC application will generate traffic depending on the active applications. This traffic must be characterized according to the properties of these applications. At this point, two main applications have been identified:

- ADS (Automatic Dependant Surveillance): this application is close to real time surveillance thanks to the use of periodical reports from the planes. An ADS report encompasses the position of the plane associated to its ICAO address.

- CPDLC (Controller-Pilot Data Link Communications): this application provides a message oriented link between pilots and air controllers. The gain expected from this data link is particularly high for transoceanic flights that rely so far on $\mathrm{HF}$ communications.

\section{B. GSM over DVB links}

The study makes the hypothesis that a GSM access network can be deployed in the cabin. The main challenge is then to define a data format (encapsulation for transmission over DVB links) and service policies. The choice of an IP or a MPEG encapsulation is still an open issue.

\section{IP traffic}

With the objective to provide an Internet access for passengers, it is of great importance to precisely define the TCP/IP architecture and the functionalities to be implemented in the different nodes of the access network:

- IP: definition of the addressing plane (NAT, DHCP...) and mobility management (re-routing of data in case of a change of earth station).

- TCP: choice of a connection management strategy (spoofing or splitting) and definition of the required functionalities of PEP (Performance Enhancement Proxy)

- Application: analysis of the offered quality of service depending on the activated options (persistent http, pre-fetching...).

\section{Convergent network}

The different traffic flows must be multiplexed on single DVB links, however differentiated services must be provided to each type of flow. Solutions using a cross-layer approach may be considered here.

\section{Conclusion}

In this paper, we have discussed an interactive system design for Internet access and ATC traffic as a first step of the $\mathrm{PhD}$ work conducted by prime author $\mathrm{Na} \mathrm{TAO}$. The preliminary design bases on ETSI DVB-S2/DVB-RCS architecture using a GEO bent pipe satellite. The requirements for both traffic types are different: multimedia traffic needs capacity and bounds on delay performance parameters; ATC traffic needs very low capacity but high reliability. Thanks to the chosen prioritizing mechanisms, both traffics can be transmitted on the same link with good performances.

The main hypothesis of the presented system design is the use of very high frequency. The use of Ka-band on the return link is favourable since bandwidth has been reserved at these frequencies for mobile services (on the contrary to $\mathrm{Ku}$ band where derogatory dispositions are needed). However, FMT (Fade Mitigation Techniques) must be activated with a major impact on resource management and eventually system performance.

Preliminary results demonstrate the technique feasibility of such system with increased capacity. More details and the open issues will be addressed in the future of this thesis work; the main objective is to demonstrate ATC, voice and Internet traffic can be converged and carried using DVB satellite links.

\section{Acknowledgment}

The authors would like to thank ONERA (Office National d'Etudes et de Recherches Aerospatiales) and more precisely the DEMR (Electromagnetism and Radar) department in Toulouse for supplying the synthesized time series for fade attenuation that have been used in this study.

\section{REFERENCES}

[1] ETSI EN 301790 v1.4.1, "Digital Video Broadcasting (DVB); Interaction channel for satellite distribution systems", 2005-09

[2] ETSI EN 302307 v1.1.1, "Digital Video Broadcasting (DVB); Second generation framing structure, channel coding and modulation systems for Broadcasting, Interactive Services, News Gathering and other broadband satellite applications", 2005-03

[3] A. Bolea-Alamanac, M. Bousquet, L. Castanet, K; Leconte, R. Rinaldo, "IFMT performance assessment on a point-to-point oriented multimedia broadband communication scenario", $22^{\text {nd }}$ AIAA International Communications Satellite Systems Conference and Exhibit (ICSSC) May 2004, Monterey (USA)

[4] ITU "Handbook on Satellite Communications Third Edition, Annex 1 propagation, AN2 signal attenuation", Wiley-Interscience

[5] RTCA Special Committee-189, ICAO Manual on Required Communications Performance (RCP Manual)

[6] EUROCAE WG 53, Guidelines for approval of the provision and use of air traffic services supported by data communications (ED-78A)

[7] L. Castanet, J. Lemorton, F. Lacoste, C. Riva, E. Mattriccianei, U.C. Fiebig, M.M.J.L. Van de Kamp, A. Martelluci, "Development and validation of time series synthesizers for Ka-band satellite communication systems", $10^{\text {th }}$ Ka-band Utilisation Conference, October 2004, Vicenza (Italy) 